
nature »: Une pratique scientifique au service de l'action (XIX $\mathrm{X}^{\mathrm{e}} \mathrm{XX} \mathrm{X}^{\mathrm{e}}$ siècles)

\title{
L'expérimentation « en plein air » ou « grandeur nature »
}

"Out-of-doors" experiments

Konstantinos Chatzis et Claudine Fontanon

\section{OpenEdition}

Journals

Édition électronique

URL : http://journals.openedition.org/dht/1615

DOI : 10.4000/dht.1615

ISSN : $1775-4194$

Éditeur :

Centre d'histoire des techniques et de l'environnement du Cnam (CDHTE-Cnam), Société des élèves du CDHTE-Cnam

\section{Édition imprimée}

Date de publication : 1 décembre 2011

Pagination : $7-14$

ISBN : 978-2-9530779-7-1

ISSN : 0417-8726

Référence électronique

Konstantinos Chatzis et Claudine Fontanon, «L'expérimentation « en plein air » ou « grandeur nature » », Documents pour l'histoire des techniques [En ligne], 20 | 2e semestre 2011, mis en ligne le 13 septembre 2012, consulté le 23 septembre 2020. URL : http://journals.openedition.org/dht/1615 DOl : https://doi.org/10.4000/dht.1615 


\title{
L'expérimentation " en plein air ॥ ou "grandeur nature "
}

Konstantinos Chatzis

IFSTTAR/LATTS

Claudine Fontanon

EHESS, Centre Alexandre Koyré (CNRS UMR 8560)

\begin{abstract}
Résumé
Placé en tête du dossier consacré à l'expérimentation " en plein air » ou " grandeur nature ", ce texte introductif poursuit un double objectif. Le premier est de nature historiographique : il s'agit de dessiner à (très) grands traits le paysage récent formé par les travaux relevant des sciences humaines et de la philosophie des sciences qui ont comme objet l'expérience scientifique - la science est prise ici au sens large du terme, les «sciences de l'ingénieur » en faisant partie. Sans viser à l'exhaustivité, il ambitionne néanmoins de dégager de la littérature existante sur la question de l'expérimentation une série de grands thèmes qui semblent avoir le plus concentré les regards et polarisé les débats. Après avoir placé l'ensemble du dossier dans un contexte plus large, nous procéderons à une présentation rapide des textes ici réunis et tenterons de tirer quelques conclusions de notre exploration collective de ce type particulier d'activité scientifique que sont les expériences " grandeur nature » ou « en plein air ».
\end{abstract}

Résumés et mots-clés en anglais sont regroupés en fin de volume, accompagnés des mots-clés français

C ommençons par un constat largement partagé. L'expérimentation' a été pendant très longtemps le parent pauvre des études sur la science. Cette déclaration peut surprendre de prime abord. II suffit, en effet, d'ouvrir n'importe quel ouvrage qui se réfère à la science post-galiléenne pour compter aisément plusieurs occurrences des termes d'expérience et d'expérimentation. Plus encore. II n'est pas rare que l'expérimentation soit explicitement placée aux fondements même de la Révolution scientifique et de la science moderne. Certes. Mais à regarder de plus près, force est de constater que dans le couple « théorie/ expérience ॥, c'est la théorie qui est privilégiée dans les faits, l'expérimentation étant alors souvent traitée comme la «servante » de celle-ci².

1 Sauf mention explicite, on utilise les termes d'expérience (scientifique) et d'expérimentation de façon invariable.

2 Le terme utilisé en anglais est celui de " handmaiden " (David C. Gooding, "Experiment ॥, dans William H. Newton-

\section{L'expérimentation vue par les philosophes des sciences} et les praticiens des sciences humaines

Pendant longtemps, les philosophes des sciences au $X X^{e}$ siècle ont le plus souvent réduit, par une sorte de glissement continu, l'acte expérimental à ses seuls résultats finaux. Faire une expérience équivaut alors à obtenir une série de données solidement établies, des « faits » non problématiques et incontestables. Réduite à son étape finale, à savoir les résultats obtenus, l'expérimentation

Smith (ed.), A Companion to the philosophy of science, Oxford, Blackwell, 2001, pp. 117-126, p. 117). Sur ce constat, voir aussi lan Hacking, "Les philosophes de l'expérience ", Tracés. Revue de sciences humaines, n 9, automne 2005, pp. 67-82 (éd. or. 1989) ; Allan Franklin, auteur d'un livre intitulé The neglect of experiment, Cambridge, Cambridge University Press, 1986, était aussi parmi les premiers à insister sur le fait que les historiens et les philosophes des sciences avaient négligé la dimension expérimentale de l'activité scientifique. 
est placée ainsi au service de la théorie. Pour les " inductivistes », comme Rudolf Carnap, les données expérimentales, patiemment accumulées, sont mobilisées pour soutenir des édifices théoriques. Pour les "déductivistes " à la Karl Popper, elles contrôlent la pertinence des théories existantes et, en cas de désaccord entre l'empirie et la théorie, elles poussent les savants à imaginer des nouveaux schémas théoriques ${ }^{3}$.

Cette primauté de la théorie sur l'expérimentation - et sur l'u observation » en général, les expériences étant souvent assimilées, par ailleurs, à une sorte d'u observation provoquée " pour parler comme Claude Bernard dans son Introduction à l'étude de la médecine expérimentale (1865) - est même renforcée avec le tournant « post-empiriste » ou « postpositiviste » qui a marqué le champ de l'épistémologie dans les années 1960 : point de donnée pure non imprégnée de théorie (" theory laden ») pour les postkuhniens ${ }^{4}$, qui contestent désormais l'autonomie, par rapport aux théories en lice, dont jouissaient jusqu'alors les observations et les données produites par l'expérimentation.

En quittant la philosophie des sciences pour les sciences humaines et sociales, force est de constater que la primauté accordée à la théorie aux dépens de l'expérimentation a affecté également pendant longtemps la communauté des historiens intéressés à l'activité scientifique. Le cas d'Alexandre Koyré est emblématique de cette tendance. Que doit Galilée à l'expérience, s'interroge le grand historien de la Révolution scientifique? Quasiment rien, répond Koyré, il n'est même pas certain, par ailleurs, qu'il ait réalisé effectivement les expériences qu'il relate dans ses

3 Karl Popper a écrit par exemple dans La Logique de la découverte scientifique (Logik der Forschung) (1934) que " la théorie commande le travail expérimental de sa conception aux derniers maniements en laboratoire ". Un demi-siècle plus tard, un autre célèbre philosophe des sciences, Bas van Fraassen, déclarait : " L'expérience, c'est la continuation de la théorie par d'autres moyens " (les deux citations sont commodément puisées dans lan Hacking, « La philosophie de l'expérience : illustrations de l'ultrafroid », Tracés. Revue de sciences humaines, n 11, 2006/1, pp. 195-228, p. 195 et p. 196, respectivement).

4 Sur ce tournant, l'ouvrage de Pierre Jacob (textes choisis, traduits et présentés par), De Vienne à Cambridge. L'héritage du positivisme logique de 1950 à nos jours, Paris, Gallimard, 1980, reste toujours très utile. C'est Norwood Russel Hanson (1924-1967) qui semble avoir forgé le premier, dans son livre Patterns of discovery (Cambridge, Cambridge University Press, 1958), I'expression " theory laden " ou " theory loaded » pour l'appliquer aux observations sensorielles. écrits ${ }^{5}$. Si le « mépris » de Koyré pour l'expérimentation n'a pas nécessairement été partagé dans son intensité par la majorité des historiens des sciences, ces derniers n'en ont pas moins privilégié dans leur pratique une histoire des sciences centrée sur la constitution et la trajectoire des concepts et des théories ${ }^{6}$, ces dernières étant conçues alors comme un système cohérent d'énoncés logiquement articulés entre eux $x^{7}$.

Le tableau que nous venons de dresser à grands traits connaîtra à partir du tournant des années 1970-1980 plusieurs mutations. Des philosophes, des praticiens des sciences sociales - dont des sociologues et des anthropologues pratiquant des enquêtes de nature ethnographique dans des laboratoires de recherche $^{8}$-, suivis bientô†

5 Sur la question de Galilée-expérimentateur, on peut se reporter commodément à Pierre Thuillier, "Galilée et l'expérimentation ॥, dans Michel Biezunski (articles choisis et présentés par), LA RECHERCHE en histoire des sciences, Paris, La Recherche/Éditions du Sevil, 1983, pp. 113-148; Paolo Palmieri, «A phenomenology of Galileo's experiments with pendulums $»$, The British Journal for the History of Science, vol. 42, n 4, 2009, pp. 479-513.

6 Le cas de Georges Canguilhem est aussi représentatif, nous semble-t-il, de cette tendance assez générale, tant en France qu'ailleurs, à privilégier la théorie. Canguilhem écrit en effet : « L'histoire des sciences peut sans doute distinguer et admettre plusieurs niveaux d'objets dans le domaine théorique spécifique qu'elle se constitue : documents à cataloguer; instruments et techniques à décrire ; méthodes et questions à interpréter ; concepts à analyser et à critiquer. Cette dernière tâche seule confère aux précédentes la dignité d'histoire des sciences " (Georges Canguilhem, "Objet de l'histoire des sciences», dans id., Études d'histoire et de philosophie des sciences, 7e éd., Vrin, Paris, 1994, p. 19 ; cité par Christian Licoppe, La formation de la pratique scientifique : le discours de l'expérience en France et en Angleterre (1630-1820), Paris, Éditions La Découverte, 1996, p. 8). lan Hacking, dans son Concevoir et expérimenter (tr. française du Representing and intervening, Cambridge, Cambridge University Press, 1983) (Paris, Christian Bourgois Éditeur, 1989, p. 249), rapporte une anecdote ayant comme auteur Thomas Kuhn, qui semble avoir fait aussi preuve, dans son travail éditorial pour le Dictionary of scientific biography, de ce préjugé en faveur de la théorie, au détriment de l'expérience. Voir aussi les bilans dressés par Davis Baird, " Internal History and the Philosophy of Experiment : An Essay Review of the Creation of Scientific Effects by Jed Z. Buchwald; Experiment and the Making of Meaning by David Gooding ; The Mangle of Practice by Andrew Pickering 11, Perspectives on science, vol. 7, n 3, 1999, pp. 383-407. $7 \mathrm{Un}$ « set of operations on propositions ॥, diraient les Anglais (D. C. Gooding, «Experiment », op. cit., p. 118).

8 Pour une revue de la première cohorte des études sociologiques et anthropologiques ayant comme objet le 
d'historiens", se sont ligués en effet depuis pour clamer par des voies différentes que l'« expérimentation a [...] sa vie propre $\|^{10}$, largement indépendante de celle de la théorie ${ }^{11}$.

laboratoire et les pratiques expérimentales qui s'y déploient (travaux de : Bruno Latour et Steve Woolgar, Michael Lynch, Karin D. Knorr-Cetina ...), voir : Karin D. Knorr-Cetina, " The ethnographic study of scientific work : towards a constructivist interpretation of science ", dans Karin D. Knorr-Cetina et Michael Mulkay (eds.), Science observed. Perspectives on the social study of science, Londres, Sage Publications, 1983, pp. 115-140. Le lecteur intéressé peut consulter aussi les analyses et jugements d'un autre auteur de cette première vague, Michael Lynch, Scientific practice and ordinary action. Ethnomethodology and social studies of science, Cambridge, Cambridge University Press, 1993, ch. 3. Pour l'état du champ des "Laboratory Studies » au milieu des années 1990 et 2000 respectivement, voir : Karin D. Knorr Cetina, "Laboratory studies. The cultural approach to the study of science "), dans Sheila Jasanoff, Gerald E. Markle, James C. Petersen et Trevor Pinch (eds), Handbook of science and technology studies, Londres, Sage Publications, 1995, pp. 140-166 ; Park Doing, « Give me a laboratory and I will raise a discipline: the past, present, and future politics of Laboratory Studies in STS ॥, dans Edward J. Hackett, Olga Amsterdamska, Michael Lynch et Judy Wajcman (eds.), The handbook of science and technology studies, Cambridge (Mass.), The MIT Press, 2007 (3e ed.), pp. 279-295.

9 Contentons-nous de signaler ici deux ouvrages publiés dans les années 1980, qui ont obtenu depuis le statut de classique : Steven Shapin et Simon Schaffer, Leviathan et la pompe à air : Hobbes et Boyle entre science et politique, Paris, Éditions La Découverte, 1993 (éd. or. 1985) ; Peter Galison, Ainsi s'achèvent les expériences. La place des expériences dans la physique du XXe siècle, Paris, La Découverte, 2002 (éd. or. 1987).

10 I. Hacking, Concevoir et expérimenter, op. cit., p. 246. Notons que l'intérêt pour l'expérimentation à la fois bénéficie (du) et alimente à son tour le renouveau profond qui a affecté l'histoire des sciences depuis le milieu des années 1980. Sur ce renouveau, voir, entre autres, la synthèse personnelle proposée par : Dominique Pestre, Introduction aux Science Studies, Paris, Éditions La Découverte, Paris, 2006 ; id., " Pour une histoire sociale et culturelle des sciences: nouvelles définitions, nouveaux objets, nouvelles pratiques ", Annales HSS, nº 3, mai-juin 1995, pp. 487-522.

11 Étant donné que ce dossier des Documents pour l'histoire des techniques réunit des contributions relevant essentiellement de la science historique, nous avons laissé de côté le questionnement épistémologique contemporain relatif à l'expérimentation. Pour un échantillon de thèmes et de questions qui animent aujourd'hui les philosophes des sciences intéressés à l'expérimentation - tels que : « la réalisation matérielle des expériences et sa signification philosophique », " expérimentation et causalité ", " les rapports entre la théorie et l'expérimentation », « expérimentation et découverte scien-
Malgré leurs différences tant au niveau des techniques d'enquête qu'à celui des objectifs poursuivis, les travaux des chercheurs qui, depuis un quart de siècle déjà, se sont penchés sur la question de l'expérience scientifique, partagent une sensibilité commune : loin de réduire l'acte expérimental à son étape ultime (les résultats finaux), ils le considèrent comme un processus long et complexe qui mérite d'être étudié dans toutes ses composantes et manifestations.

Expérimenter, nous disent ces chercheurs, est, en effet, un véritable travail qui mobilise plusieurs acteurs, pris dans des relations multiples et variées. Il y a d'abord les expérimentateurs, des êtres en chair et en os, qui raisonnent " en situation de travail $\|^{12}$, qui manipulent la matière grâce à des savoirs et des savoir-faire en grande partie tacites $^{13}$, qui communiquent et interagissent intensément entre eux - de façon informelle à l'intérieur de leur espace commun de travail mais aussi à distance grâce au pouvoir de l'écrit -, qui s'engagent dans des controverses qui s'avèrent souvent difficiles à terminer ${ }^{14}$, qui relèvent de

tifique ", "la signification philosophique des instruments »... -, voir, par exemple, Hans Radder (ed.), The philosophy of scientific experimentation, Pittsburgh, University of Pittsburgh, 2003 ; Friedrich Steinle, « Experiments in history and philosophy of science $॥$, Perspectives on science, vol. 10, n 4, 2002, pp. 408-432 ; Allan Franklin, « Experiment in physics », Stanford encyclopedia of philosophy, publié le 5 octobre 1998, révisé le 6 janvier 2009, disponible à l'adresse : http://plato.stanford. edu/entries/physics-experiment/ et consulté le 4 janvier 2012.

12 Voir, par exemple, le travail pionnier de Karin D. Knorr-Cetina, The manufacture of knowledge, Oxford, Pergamon Press, 1981. 13 Pour la reconnaissance de l'importance des savoirs et savoir-faire tacites dans la production et la diffusion de la connaissance scientifique, les travaux de Harry Collins et d'Otto Sibum ont été décisifs. Voir par exemple : Harry Collins, Changing order : replication and induction in scientific practice, Londres, Sage Publications, 1985 ; Trevor Pinch, Harry M. Collins et Larry Carbone, « Inside knowledge : second order measures of skill "), The sociological review, vol. 44, n², 1996, pp. 163-186; H. Otto Sibum, " Les gestes de la mesure. Joule, les pratiques de la brasserie et la science 1), Annales HSS, $n^{\circ} 4-5$, juillet-octobre 1998, pp. 745-774. Voir aussi Simon Schaffer, "Experimenters' techniques, dyers' hands and the electric planetarium ", Isis, vol. 88, n³, 1997, pp. 456-483.

14 La question de la controverse (et de sa clôture) ainsi que celle de l'achèvement d'une expérience scientifique sont au centre de plusieurs travaux consacrés à l'expérimentation. Voir, entre autres: Harry Collins et Trevor Pinch, Tout ce que vous devriez savoir sur la science, Paris, Éditions du Seuil, 1994 (1 1 ère éd. $1993)$; S. Shapin et S. Schaffer, Leviathan et la pompe à air..., op. cit. ; P. Galison, Ainsi s'achèvent les expériences..., op. 
multiples univers institutionnels et sociaux et qui évoluent au long de plusieurs réseaux de sociabilité ...

Mais l'expérimentateur, seul ou en compagnie de ses collègues au sein d'équipes qui peuvent atteindre des tailles impressionnantes au fur et à mesure qu'on avance dans le temps, n'est pas le seul acteur de la scène expérimentale : des protecteurs ${ }^{15}$, des financeurs, des constructeurs d'instruments, des ingénieurs-concepteurs de grands dispositifs techniques, sans oublier les différents publics qui assistent aux expériences ${ }^{16}$, peuplent aussi la scène expérimentale dans des proportions qui varient selon les époques et les disciplines.

Eł ce n'est pas tout. À côté des humains prend également place toute une pléiade d'êtres inanimés: dans les sites, historiquement variables où est pratiquée l'expérimentation - le cabinet du savant, le laboratoire moderne ... -, on trouve en effet des tubes et des flacons, des échantillons, des calculateurs, des appareils de mesure et de contrôle, des instruments d'observation et d'enregistrement ${ }^{17}$, toutes sortes d' " inscriptions littéraires $1{ }^{18}$ enfin, telles que des diagrammes et des graphiques, des chiffres archivés, des carnets de note, des articles et des livres...

cit. ; Peter Machamer, Marcello Pera et Aristides Baltas (eds.), Scientific controversies. Philosophical and historical perspectives, New York, Oxford University Press, 2000 ; H. Tristram Engelhardt Jr et Arthur L. Caplan (eds.), Scientific controversies: case studies in the resolution and closure of disputes in science and technology, New York, Cambridge University Press, 1987. 15 Sur ce sujet, voir en premier lieu le désormais "classique " (et controversé) livre de Mario Biagioli, Galieo Courtier: The Practice of Science in the Culture of Absolutism, Chicago, The University of Chicago Press, 1993.

16 Voir, par exemple, Steven Shapin, "The house of experiment in seventeenth-century England 1", Isis, vol. 79, n³, 1988, pp. 373-404 (l'auteur met en évidence un public de « gentlemen »).

17 Albert von Helden et Thomas L. Hankins (eds.), "Instruments », Osiris, vol. 9, 1994 ; Frederic L. Holmes, Trevor $\mathrm{H}$. Levere (eds.), Instruments and experimentation in the history of chemistry, Cambridge (Mass.), The MIT Press, 2000 ; Marie-Noëlle Bourguet, Christian Licoppe et H. Otto Sibum (eds.), Instruments, travel and science: itineraries of precision from the seventeenth to the twentieth century, Londres, Routledge, 2002 ; Davis Baird, Thing Knowledge: A philosophy of Scientific Instruments, Berkeley et Los Angeles, University of California Press, 2004.

18 Pour une vision du laboratoire (et de l'activité expérimentale qui s'y déploie) comme un lieu de production d'inscriptions littéraires, voir le désormais classique Bruno Latour et Steve Woolgar, La vie de laboratoire. La production des faits scientifiques, Paris, La Découverte/Poche, 1996 (1 lère ed. 1979).
Restons un peu sur ce dernier type d' «inscription littéraire ». Plusieurs travaux récents montrent que loin d'être des dépositaires passifs d'un travail fait ailleurs (dans les seuls laboratoires, par exemple), livres et articles sont des acteurs du travail expérimental à part entière : en tant que dispositifs conçus pour convaincre tous ceux qui n'avaient pas assisté physiquement à l'expérience ${ }^{19}$, ou en tant que machines à transformer, grâce à une chaîne d'opérations, des énoncés "locaux "portant sur des mesures obtenues à un moment donné à l'aide d'un instrument spécifique manié par un individu particulier, par exemple - en des énoncés scientifiques de validité universelle, des "faits" acceptés par tous sans discussion ${ }^{20}$.

Même menée au pas de course, cette excursion au sein de la galaxie formée par les recherches portant sur l'expérimentation met en évidence la vivacité de ce champ d'études ${ }^{21}$. Le présent dossier des Documents pour l'histoire des techniques veut à la fois profiter de cette effervescence autour du sujet et apporter sa propre contribution à la galaxie précitée. Eł ceci en dirigeant le regard sur une région peu explorée jusqu'à présent : les expériences faites en dehors des murs protecteurs du laboratoire ${ }^{22}$.

19 Sur ce thème, voir S. Shapin et S. Schaffer, Leviathan et la pompe à air..., op. cit. On doit à Steven Shapin l'expression de "technologie littéraire " pour désigner les moyens par lesquels une expérience est communiquée à ceux qui n'en avaient pas été le témoins directs (voir : Steven Shapin, "Pump and circumstances : Robert Boyle's literary theory ", Social studies of science, vol. 14, $n^{\circ} 4,1984$, pp. 481-520).

20 Le classique ici est B. Latour et S. Woolgar, La vie de laboratoire..., op. cit.

21 Pour une série de références, autres que celles déjà citées, on peut consulter les bibliographies des articles et ouvrages suivants : Jeffrey L. Sturchio, " Artifact and Experiment I, Isis, vol. 79, n³, 1988, pp. 368-372 ; Dominique Vinck, "Retour sur le laboratoire comme espace de production de connaissances "I, Société d'anthropologie des connaissances/Revue d'anthropologie des connaissances, $\mathrm{n}^{\circ} 2$, 2007/2, pp. 159-165 ; D. C. Gooding, "Experiment », op. cit. ; K. Knorr Cetina, "Laboratory Studies... ", op. cit. ; P. Doing, "Give me a Laboratory... ", op. cit. ; H. Radder (ed.), The philosophy..., op. cit. ; D. Pestre, Introduction..., op. cit. ; id., "Pour une histoire sociale... », op. cit. ; Peter Galison, Image and Logic : A Material Culture of Microphysics, Chicago, The University of Chicago Press, 1997 ; Max-Planck - Institut für Wissenschaftsgeschichte, The shape of experiment, Actes d'une conférence tenue à Berlin, 2-5 juin 2005, Preprint n 318, 2006, disponible à l'adresse : http://www.mpiwg-berlin.mpg.de/ Preprints/P318.PDF (consulté le 4 janvier 2012).

22 Sur le thème des sciences pratiquées en dehors des murs du laboratoire, voir la collection d'articles dans Henrika 


\section{La composition du numéro}

C'est dans le cadre de notre séminaire de l'EHESS consacré depuis l'année 2005 aux productions et pratiques scientifiques des ingénieurs français, civils ou militaires, dans la France du $X I X^{e}$ siècle et début du $X X^{e}$ siècle que le thème des expériences "en vraie grandeur " ou "en plein air " a émergé. Plusieurs communications montraient qu'il existait une documentation abondante mais quasi inexplorée, produite par ces ingénieurs soucieux d'apporter des solutions rationnelles aux problèmes que posaient au cours de cette longue période, l'amélioration des réseaux de communications, l'exploitation des ressources du territoire national, l'aménagement urbain ou le réaménagement des établissements d'État, manufactures et arsenaux. Mais peu d'historiens de sciences avaient jusqu'ici reconnu le caractère scientifique de ces rapports d'expériences, bien que la plupart des ingénieurs eussent revendiqué ce statut pour leurs écrits, en communicant régulièrement, par exemple, leurs résultats à l'Académie des sciences. Nous avons donc inscrit cette thématique à l'ordre du jour de notre séminaire et mobilisé parmi les historiens des sciences et des techniques ceux dont les recherches croisaient notre problématique. II en résulta sept communications particulièrement pertinentes qu'il nous a semblé impératif de publier pour apporter une contribution que nous pensions originale à l'historiographie sur l'expérience scientifique, exposée brièvement dans la première partie de I'Introduction au dossier.

Le présent dossier proposé au lecteur des Documents pour l'histoire des techniques est le résultat de ce travail collectif mené au cours des années 2005 et 2006. A travers leurs recherches, les sept auteurs nous montrent la richesse des corpus documentaires mobilisés, la variété des dispositifs expérimentaux imaginés par les ingénieurs et la diversité des finalités de ces pratiques scientifiques au service de l'action.

À travers les expériences d'hydraulique réalisées en plein air et en vraie grandeur par les officiers du génie Jean-Victor Poncelet et JosephAimé Lesbros, Bruno Belhoste entend analyser une tradition expérimentale originale dont les méthodes sont très différentes de celles des physiciens expérimentateurs, beaucoup plus étudiées par

Kuklick et Robert E. Kohler (eds.), "Science in the field", Osiris, vol. 11, 1996. les historiens des sciences. L'historiographie $a$, en effet, mis l'accent sur le laboratoire, et l'auteur souhaite consacrer son article à une autre modalité expérimentale avec d'autres types d'acteurs et d'autres lieux (un chantier, une route, un fleuve, un champ de bataille...), pour souligner l'importance et la vitalité d'autres cultures expérimentales, en l'occurrence celle spécifique aux ingénieurs hydrauliciens entre 1750 et 1850. Bruno Belhoste commence par préciser la dimension théorique et expérimentale de l'écoulement de l'eau par un orifice (loi de Toricelli, observations de Newton et de Mariotte, expériences de Borda, de Venturi, de Bossut, de Michelotti et de Hachette) puis détaille l'organisation des expériences réalisées par Poncelet et Lesbros en 1827 et 1828. L'auteur explique que pour l'ordonnateur de ces expériences, le général Sabatier, commandant de l'École de Metz, il s'agit de prolonger les expériences entreprises à Mézières au XVIII siècle par Dubuat et ainsi de rehausser le prestige de l'École de Metz, alors que pour Poncelet le but est de compléter ses recherches sur les dépenses d'eau menées à l'occasion de ses travaux sur les roves hydrauliques - Poncelet souhaite alors établir des tables de dépense d'eau pouvant servir à la construction et au fonctionnement de machines hydrauliques et des écluses. Après avoir décrit avec précision les modalités de l'expérience létalonnage du bassin de jauge, réglage de l'ouverture de l'orifice et de la hauteur d'eau du réservoir...), Bruno Belhoste s'intéresse à la diffusion et à la réception du mémoire des deux ingénieurs, publié par l'Académie des sciences en 1832. ॥ insiste sur les limites de ce travail expérimental qui selon lui, n'aboutit qu'à perfectionner les résultats déjà obtenus, et n'apporte aucun résultat notable à la connaissance des lois de l'écoulement. Ce qui explique que le mémoire soit tombé dans l'oubli chez les historiens de l'hydraulique et de la mécanique des fluides. Toutefois, l'auteur signale qu'à l'occasion de ces expériences, Poncelet a découvert incidemment le phénomène des "rides capillaires " qui sera théorisé en 1871 par Thomson. En dépit de ce constat décevant, Bruno Belhoste conclut à l'évolution profonde des conditions de l'expérimentation au début du XIXe siècle, les expériences de Poncelet et de son collaborateur ouvrant un nouveau front - les expériences de mécanique en vraie grandeur - qui fournit un modèle aux ingénieurs et expérimentateurs hydrauliciens en Europe et aux États-Unis.

Martin Zerner, historien des sciences (CNRS/ Université Paris Diderot-Paris VII) a analysé l'œuvre scientifique de l'ingénieur des ponts et chaussées, Henri 
Darcy (1803-1856) dans le domaine de l'hydraulique. L'auteur s'est penché, en particulier, sur le livre monumental publié par Darcy en 1856 sur Les fontaines publiques de la Ville de Dijon, dans lequel l'ingénieur expose pour la première fois la loi, qui porte par ailleurs son nom, sur l'écoulement de l'eau à travers le sable et, plus généralement, celui d'un fluide à travers un milieu poreux. Pourtant, tenter d'établir une loi serait, selon l'auteur, une démarche naturelle selon la culture scientifique des ingénieurs de l'époque, aussi l'auteur de l'article focalise-il son analyse sur la genèse de l'intérêt de Darcy pour les questions hydrauliques et sur les expériences menées dans ce domaine depuis l'époque de son affectation, sous la Restauration, à Dijon, où il étudie un projet d'adduction d'eau dans la ville. C'est dans les années 1830 que naît I'intérêt de Darcy pour les puits artésiens dont il mesure le débit, et c'est à Paris, en 1848, où il est nommé directeur des services techniques de la ville que l'ingénieur des ponts met en chantier ses expériences sur le mouvement des eaux dans les tuyaux, publiées en 1857 sous forme de mémoire adressé à l'Académie des sciences. Pour ces expériences, Darcy dispose, par ailleurs, de moyens considérables, car l'objectif assigné à ces travaux par le corps des ponts et chaussées est de fournir aux ingénieurs chargés des adductions d'eau un moyen fiable de calcul du débit en fonction de la charge hydraulique. L'auteur nous explique que les expériences et mesures effectuées à chaque occasion par Darcy portent sur des situations rencontrées couramment dans la pratique des ingénieurs, mais que l'ingénieur en a profité pour étudier la répartition des vitesses à l'intérieur des tuyaux et effectuer quelques mesures pour les faibles vitesses. C'est grâce à ces mesures qu'il établit une nouvelle loi purement expérimentale en 1851 qui sert de fondement à la loi de Darcy évoquée plus haut. Notons que cette loi est encore aujourd'hui à la base des calculs des ingénieurs dans de multiples domaines (extraction du pétrole, enrichissement de l'uranium ...).

Konstantinos Chatzis (IFSTTAR/LATTS) expose les termes de la controverse qui oppose deux polytechniciens, Jules Dupuit et Arthur Morin, dans les années 1830/1840 sur la question du "frottement de roulement ". Mais plutôt que de détailler le contenu de la controverse (régimes d'argumentation et administration de la preuve), l'auteur choisit de camper la scène scientifique française et de proposer, par l'intermédiaire de la controverse, une anthropologie historique du savant (français) de l'époque. L'article démarre avec un exposé succinct des enjeux économiques et politiques liés à l'instauration d'une police réglementant la circulation routière (" la police du roulage ") et de ses conséquences pratiques pour l'art de l'ingénieur ; question d'autant plus brûlante que ces années sont marquées par des programmes routiers de grande ampleur. L'auteur détaille, ensuite, les expériences "grandeur nature " menées en province indépendamment par l'ingénieur des ponts Dupuit et par l'artilleur Morin sur le frottement de roulement, et présente les résultats divergents auxquels sont parvenus les deux expérimentateurs. Alors que Morin obtient les moyens de poursuivre ses expériences et Dupuit l'autorisation du corps des ponts et chaussées d'effectuer de nouvelles recherches, la scène scientifique devient parisienne et placée sous le contrôle de l'Académie des sciences et d'une commission des ponts et chaussées, sollicitées par les deux rivaux. Puis l'auteur s'intéresse à la « mise en texte » du travail expérimental (rapports, essais, articles) et à ce qu'il désigne après Steven Shapin sous le vocable de "technologies littéraires", caractérisant les moyens d'exposition des résultats obtenus par la voie expérimentale. L'auteur conclut sur le caractère éminemment pratique des préoccupations des deux polytechniciens, désireux d'informer les pratiques d'un État qui se veut de plus en plus rationnel, et sur le couplage, ici direct, entre "science fondamentale " et action, pour finalement s'interroger sur la subjectivité du savant engagé dans la controverse scientifique.

Claudine Fontanon (EHESS/Centre Alexandre Koyré) s'est intéressée aux expériences d'hydrodynamique effectuées par l'ingénieur de l'artillerie Arthur Morin dans le cadre de la commission dite " des principes du tir » crée en 1833 par le ministère de la Guerre. Bien qu'il s'agisse pour cette commission de réfléchir à l'amélioration de la formation des élèves des écoles d'artillerie, Arthur Morin et deux ingénieurs qui lui sont associés, Guillaume Piobert et Isidore Didion, décident d'élargir le programme de recherches militaires qui leur est soumis aux questions fondamentales, et non résolves à l'époque, de la résistance des différents milieux, notamment l'air et l'eau, à la pénétration des projectiles. Après avoir situé les trois acteurs de la commission des principes du tir par le biais de courtes biographies, l'auteur évoque les circonstances qui ont amené Arthur 
Morin à effectuer des séries d'expériences « en plein air ॥ entre 1836 et 1841 sur la résistance de l'eau à l'avancement des corps immergés aux petites et grandes vitesses, puis semi-immergés, enfin à l'avancement des corps flottants. II s'agit pour l'expérimentateur de préciser expérimentalement les coefficients de la loi de la résistance de l'eau à l'avancement des corps donnée par Newton aux différentes vitesses. Les expériences concernant les corps flottants entreprises à Metz et poursuivies dans les environs de Paris, sur le canal de l'Ourca puis sur le canal Saint-Denis, sont conduites avec le souci constant des applications possibles dans le champ économique : notamment l'amélioration des formes des bateaux destinés à la navigation fluviale, en plein essor au cours des années 1830.

Georges Ribeill (LATTS) propose d'analyser les raisons pour lesquelles les essais et mesures " grandeur nature " ont progressivement pris le relais des approches théoriques "en chambre " et de la modélisation dans la conception et l'exploitation des chemins de fer. L'auteur insiste sur le fait qu'au XIXe siècle les chemins de fer ont constitué pour les ingénieurs un objet propice à la modélisation cinématique ou mécanique, en dépit des caractéristiques d'un système soumis à des perturbations d'origine environnementale, technique ou humaine. II rappelle les principales directions de ces premières recherches ferroviaires - géométrie de la voie, résistance des trains à la traction -, tout en insistant sur les limites de cette modélisation sur les questions du déraillement et de la stabilité rail/rove. Les ingénieurs ferroviaires sont alors incités à mettre en œuvre dès la fin du XIXe siècle des recherches expérimentales "grandeur nature " : dans un premier temps sur des circuits d'essais spéciaux, puis avec des trains expérimentaux circulant sur des voies ordinaires pour des essais de vitesse notamment, avec par exemple les «rodéos ferroviaires » entre Montereau et Sens, où la vitesse de $144 \mathrm{~km} /$ heure, éphémère record de vitesse en 1890, est atteinte. L'auteur aborde ensuite la question de "l'équation des freins " imposée aux réseaux français en 1901. On assiste alors à une inflation des systèmes de freins continus automatiques testés sur chaque réseau jusqu'à la conférence internationale pour l'unité technique à Berne en 1907. II en ressortira, en 1909, la rédaction d'un protocole expérimental applicable à tous les réseaux : en France le frein Westinghouse fut mis à l'essai par le PLM en 1912 ; en Allemagne, c'est le système Kunze-Knorr. Mais aucune normalisation n'aboutit avant la Première guerre mondiale. L'auteur conclut pour le XXe siècle à la tendance des ingénieurs de continuer à recourir aux modèles et aux pratiques de modélisation malgré les progrès liés aux essais. II certifie toutefois que bon nombre de progrès dans la connaissance ferroviaire résultent de méthodes de mesure " embarquées » considérablement améliorées.

Jean-Louis Bordes, historien et ingénieur des arts et manufactures, propose une étude sur la longue durée de la méthode expérimentale appliquée à l'auscultation et à la surveillance des barrages, les sites de ces ouvrages constituant selon lui un objet permanent d'essai " en vrai grandeur "I. II se propose de retracer l'histoire des appareils d'auscultation mis en œuvre au cours de procédures de plus en plus rigoureuse de surveillance de ces ouvrages d'art, exceptionnels par leurs dimensions et leur durée, en liaison avec l'évolution des techniques de construction. On peut retenir comme exemples les fissuromètres et les inclinomètres, utilisés aux États-Unis dans les années 1920 pour étudier le comportement mécanique des barrages-voûtes, ou encore le capteur à corde vibrante de l'ingénieur André Coyne conçu en 1931. L'auteur insiste sur le fait que ce sont les ingénieurs du génie civil qui ont été amenés à concevoir et construire ces instruments dans leurs tentatives de comprendre le comportement de ces grands ouvrages et d'en assurer la sécurité. Mieux, auscultation et surveillance des barrages deviendraient en France des outils de gestion du patrimoine de l'infrastructure du pays.

Benoît Lelong (LATTS, université de Paris-Est) propose d'étudier les formes de coopération entre savants, expérimentateurs et militaires à propos des appareils ultrasonores, de la Grande Guerre à la fin des années 1920. En confrontant plusieurs technologies et configurations nationales, les marines française et anglaise, l'auteur entend montrer le caractère local et négocié des formes de coordination entre laboratoires et essais en mer. A travers l'exemple du laboratoire du Centre d'études de Toulon, Benoît Lelong met en évidence les difficultés pour les chercheurs et inventeurs, d'une part, les officiers ou ingénieurs militaires, de l'autre, à travailler ensemble. II s'intéresse particulièrement à la mobilisation scientifique de 1915-1918 en France, notamment à travers les recherches réalisées par le physicien Paul Langevin, en association avec l'inventeur russe Constantin Chilowski, sur la détection sous marine par ultrasons, travaux effectués sans problèmes d'accès aux lieux de mise à l'épreuve 
de ces technologie, du laboratoire à l'essai en mer. L'auteur introduit ensuite une comparaison avec la mobilisation scientifique en Angleterre, où les travaux sont menés par de multiples groupes exploitant des expertises locales. II s'intéresse enfin à la diversification en France des formes de I'innovation suite à la réorganisation de 1921, les modalités de coordination adoptées devenant très variables selon les technologies développées dans le domaine du sondage - sondeurs ultra sonores, matériels d'écoute, microphones électromagnétiques -, et la configuration d'acteurs en présence. II recourt de nouveau à une comparaison avec l'Angleterre, en insistant sur l'importance stratégique de la détection sousmarine (les "asdics " ou sonars anglais), pour laquelle la lutte contre les sous-marins revêt une importance politique, I'Asdic Group représentant, selon l'auteur, une forme de vie scientifique sans équivalent en France, dans la mesure où elle intègre dans un même régime de pratiques des activités dispersées en France (des expériences de laboratoire aux essais en mer).
Tout en invitant le lecteur à découvrir par luimême la teneur de chaque texte et à construire ses propres vues synthétiques, soulignons de notre part la place décisive qu'occupent les ingénieurs dans ce type d'expérimentation en plein air ou en vraie grandeur, qui allie production de connaissances relevant souvent de "sciences fondamentales » et utilité pratique. Notons aussi les difficultés particulières que pose ce type d'expérimentation, à cause de multiples aléas qui interviennent lors du déroulement de l'expérience, qui ne bénéficie pas des mûrs protecteurs du laboratoire. Rien d'étonnant alors à ce que les résultats obtenus à l'occasion de ce genre d'expérimentation soient souvent difficilement reproductibles et comparables entre eux, affectés qu'ils sont par les conditions locales dans lesquelles se sont déroulées les expériences qui leur ont donné naissance. 